\title{
Reacting to Unexpected Losses in an Uncertain World: High Approach Individuals
}

\section{Become Even More Risk-seeking}

\author{
Xiaoyue $\operatorname{Tan}^{\mathrm{a}}$, Msc \\ Jan-Willem Van Prooijen ${ }^{\mathrm{a}}$, PhD \\ Travis Proulx ${ }^{\mathrm{b}, \mathrm{d}}, \mathrm{PhD}$ \\ Haizheng $\mathrm{Wu}^{\mathrm{c}}$, Msc \\ Ilja Van Beest ${ }^{\mathrm{b}}, \mathrm{PhD}$ \\ Paul A. M. Van Lange ${ }^{\mathrm{a}}$, PhD
}
${ }^{a}$ Department of Experimental and Applied Psychology, VU University Amsterdam, Amsterdam 1081 BT, The Netherlands

${ }^{\mathrm{b}}$ Department of Social Psychology, Tilburg University, PO Box 90153, 5000LE, Tilburg, The Netherlands

${ }^{\mathrm{c}}$ Department of Social and Organizational Psychology, Twente University, Enschede, The Netherlands

${ }^{\mathrm{d}}$ School of Psychology, Cardiff University, Tower Building, 70 Park Place, Cardiff, CF10 3AT, UK

\section{Word Count:}

\section{Authornote}

The authors declare that there are no potential conflicts of interest with respect to the research, authorship, and/or publication of this article. 


\begin{abstract}
Previous research has shown that people are risk-seeking in the face of losses. We propose that this risk-seeking orientation is a palliative approach response to deal with a discrepancy between people's desires of having no losses versus the possibility of loss. An expectancy violation (which induces behavioral approach responses) would therefore strengthen people's risk-seeking in the context of losses. Two experiments (Study 1 and Study 2) which were conducted in the context of the Asian Disease Problem demonstrated that people high in trait behavioral approach (trait BA) were more risk-seeking following an expectancy violation (state BA) than in a control condition. As expected, this was only the case for decisions framed in terms of losses, but not in terms of gains (Study 1). Taken together, our findings highlight the interacting motivational influences of situation-induced state behavioral approach and trait behavioral approach in understanding risky decision-making in the face of losses.
\end{abstract}

Keywords: risk taking, losses, behavioral approach, expectancy violation 


\section{Introduction}

A Chinese family of 7 members - who were not able to swim — drowned in a reservoir one after another when trying to rescue a child who fell into the water by accident. The strong motivation to save a family member in danger is completely understandable. In this case, however, the loss of life could have been significantly reduced if the family members thought twice before their actions. People are risk averse in general, but why do people make such risky decisions when confronted with possible losses? In the present study, we explore the general question: Why do people become risk-seeking in the face of losses? We focus in particular on Behavioral Approach (BA), representing a system sensitive to signals of reward, non-punishment and escape from punishment (Gray, 1990; Carver \& White, 1994; Elliot \& Thrash, 2002). BA is understood as both a trait - that is, individual differences in the trait BA (Carver \& White, 1994) — as well as a motivational state that can be situationally induced by anxious uncertainty arising from an expectancy violation, that is, experiences that are not consistent with people's beliefs or goals (McGregor, et al., 2010; Proulx, Inzlicht, \& HarmonJones, 2012). In the present research, we examine how trait and state BA influences risk seeking in the context of losses.

Even though people are found to be risk averse in general (Schneider \& Lopes, 1986), some people are more likely than others to take risks in social decision-making, in the sense that they favor a risky option with uncertain outcomes over a more secure option. Sometimes, it is necessary and reasonable to take some risks in order to improve the chances for a better life. At other times, however, some risk-taking behaviors are irrational and costly. For example, the impossible rescue attempt from the opening paragraph-which involved jumping in a deep-water reservoir without being able to swim—presumably was carried out without careful consideration of the expected costs and the likelihood of success. Furthermore, some studies have also found a similar phenomenon called "loss chasing"- the 
continued gambling to recover previous losses—among pathological gamblers (CampbellMeiklejohn, Woolrich, Passingham, \& Rogers, 2008). Therefore, it is important to discover the factors that contribute to people's irrational risk-taking decisions in the face of losses.

Several theories have been developed to understand the underlying psychological mechanisms for risk-seeking behavior in the face of losses. These theories have emphasized either the "cold" cognitive and information processing factors that shape risk preferences (for a review, see Kühberger, 1997) or the "hot" motivational factors that drive people to make risky decisions in the context of losses (Baumeister, 2003; Lopes, 1987; Scholer, et al., 2010). In the present studies, we propose that because the experience of a loss is often unwanted, unexpected and potentially threatening, people's responses towards losses are driven by a defensive approach motivational state. In particular, we argue that expectancy violation and people's trait behavioral approach play an important role in their reactions towards losses. Our research findings would extend the motivational accounts for people's risk seeking in reacting towards losses.

\section{1. "Cold" versus "Hot" theories in explaining people's risk seeking under losses}

Among the "cold" cognitive theories that address people's risk seeking under losses, prospect theory (Kahneman and Tversky, 1979) is most well-known. This theory focuses on people's perception of decision problems and the evaluation of probabilities and outcomes. According to this theory, people are loss-averse, meaning that they typically exhibit greater sensitivity to losses than to gains. As a consequence of such loss-aversion, people favor a risky option that offers the probability to restore the status quo, while people prefer a more secure option for the same problem in the context of gains. In other words, loss aversion drives people towards more risky decision-making under losses.

Meanwhile, "hot" motivational theories that emphasize the importance of motivations to account for people's risk seeking under losses have been proposed as complements to "cold" 
cognition (Lopes, 1987; Scholer, et al., 2010). For example, Lopes (1987) has emphasized that the underlying motives to achieve security (i.e., avoidance motivation) or to explore potential (i.e., approach motivation) predispose people to different orientations in response to risks, such that "potential-oriented" individuals are generally risk-seeking, whereas "securityoriented" individuals are generally risk-averse. People's risky decision-making is a result of interaction of these motives with a situational factor called "aspiration level", which reflects opportunities at hand as well as constraints imposed by the environment. According to this theory, potential-motivated individuals are more likely to take risks under losses, because the risky option offers opportunity of achieving a non-loss state which can satisfy their potentialseeking motives.

In line with these motivational mechanisms, we propose that behavioral approach motivation, which is sensitive to positive signals, plays an important role in affecting people's risk seeking under losses. We intended to investigate the motivational roles of expectancy violation, which would induce people into a behavioral approach state, together with people's trait behavioral approach orientation, in affecting people's risk-seeking under losses.

\subsection{Expectancy violation, approach motivation and risk-seeking}

People frequently have experiences that are inconsistent with their beliefs or goals. Such experiences are called expectancy violations, given that they are inconsistent with abstract mental representations of expected relations (Proulx \& Inzlicht, 2012). When people's expectations are violated, they experience a common syndrome of aversive arousal, which motivates them to engage in a variety of palliative compensatory efforts (for a review, see Proulx, Inzlicht, \& Harmon-Jones, 2012). Some scholars consider expectancy violations as threatening, and suggest a common motivational process underlying people's reactions to threats (Jonas et al., 2014). It is stated that when facing threats, for a quite short period, the 
Behavioral Inhibition System (BIS; Gray \& McNaughton, 2000) is activated, which comes together with various symptoms, such as anxious arousal, to orient organisms to solve the discrepancy. Over time, however, the Behavioral Approach System (BAS) mutes the BIS and down-regulates anxiety automatically arising from threats. Usually the approach-oriented reactions, risk taking for instance, are merely palliative because they cannot directly resolve the violation. Meanwhile, people who are disposed by behavioral approach motivation would flip to these reactive approach actions more quickly (McGregor, et al., 2010; Jonas, et al., 2014).

Whereas expectancy violation pertains to situationally induced behavioral approach motivation, previous research suggests that behavioral approach orientation is central to many personality models (Gray, 1990; Carver \& White, 1994; Elliot \& Thrash, 2002). There are studies indicating a positive relationship between trait approach orientation and risk-seeking behaviors. For example, subscales of the behavioral approach trait inventory are positively related to increased risk-seeking in the Iowa Gambling Task (IGT) (Suhr \& Tsanadis, 2007). As mentioned before, potential-oriented individuals are generally risk seeking under losses (Lopes, 1987). On the basis of these findings, the present authors emphasize the influences of BA (both trait and state) on people's risk seeking in the context of losses.

\subsection{The present research}

Based on the idea that people's risk seeking under losses is driven by a reactive behavioral approach state, we expect that after an expectancy violation-which is generally assumed to induce a behavioral approach state (McGregor, et al., 2010; Jonas, et al., 2014) people will become more risk seeking (Hypothesis 1). We also propose that there will be a positive relationship between people's risk seeking under losses and their trait behavioral approach motivation (Hypothesis 2). Given that individual differences in dispositional behavioral approach is an important moderator that determines how quickly people flip to 
approach-oriented reactive actions (Jonas, et al., 2014), we propose that those high in trait BA would be especially likely to respond to an expectancy violation in comparison to those low in trait BA. As manifested in the present research, this would lead to a heightened preference for risk for those people who are high in trait BA in the expectancy violation condition as compared to the control condition (Hypothesis 3).

The Asian disease problem (ADP) is a classic research setting to investigate loss aversion. The typical result is that people are risk seeking when outcomes are framed as losses, while they are risk averse when outcomes are framed as gains (Tversky \& Kahneman, 1981; Kühberger, Schulte-Mecklenbeck \& Perner, 1999). In this task, people are presented with a hypothetical situation whereby 600 people may die from a disease outbreak. In both the gains and losses-framed version of the task, people are asked to choose between a "sure" (a certain number survive) and "risky" (a smaller chance that nearly all survive) option in administering treatment, although the expected survival rates for each option are identical across framings. The only difference between these framings is the language used in presenting the choice options: in the gains framing, the decision is made in terms of the number of people who will "be saved." and in the loss framing, the decision is made in terms of the number of people who "will die.".

In the present research, we operationalized people's risk-seeking under losses by relying on the loss-framed Asian disease problem (ADP). Before presenting the Asian disease problem, participants either viewed a group of natural upside-down faces in a control condition or a group of anomalous upside-down faces (i.e., the Thatcher Illusion, Lewis \& Johnston, 1997) after the first three natural upside-down faces in an expectancy violation condition. In Study 1, we also included the gain-framed Asian disease problem to replicate the framing effect. In addition, because people are risk-averse instead of risk-seeking in the context of gains, we expected a three-way interaction such that the two-way interaction of 
expectancy violation and dispositional BA would only materialize under losses framing and not under gains framing (Hypothesis 4).

\section{Study 1}

In Study 1, participants were presented with either a gain or loss-framed version of the Asian disease problem (Kahneman \& Tversky, 1979). We assessed trait behavioral approach with BAS scale (Carver \& White, 1994) and manipulated expectancy violation to induce anxious uncertainty and state behavioral approach.

\subsection{Method}

\subsubsection{Participants and Design.}

201 complete responses were collected online using MTurk (\$ 0.50 compensation). 194 cases $\left(53.1 \%\right.$ female; $M_{\mathrm{age}}=38.13, S D=13.28 ; 97.9 \%$ English native speakers $)$ were deemed valid cases after excluding 7 participants who reported that they did not complete the experiment without distractions. We used a trait behavioral approach (continuous) $\times$ framing (gains versus losses) $\times$ expectancy violation (with versus without violation) design.

\subsubsection{Procedure.}

Participants were provided with an online link to the experiment presented with Qualtrics survey software. The experiment was presented as a study on the relationship between personality and ability to understand facial expressions. Participants provided demographic information, and completed a series of filler questionnaires followed by a 13-item behavioral approach scale (Carver \& White,1994, $\alpha=.87$ ). Two example items are "I go out of my way to get things I want." and "I'm always willing to try something new if I think it will be fun.". Item scale ranged from 1 (very true for me) to 4 (very false for me).

Next, participants were randomly assigned to the Control or Expectancy Violation conditions, where they viewed photos of inverted faces (original faces were obtained from Wallhoff, 2006) and were instructed to determine whether the photo expressed generally 
"positive" or "negative" emotions. In the "expectancy violation" condition, elements of the facial features constituted an implicit visual anomaly (e.g., Sleegers, Proulx \& Van Beest, 2015), whereby the eyes or mouth were oriented incorrectly relative to the general orientation of the face (i.e., the "Thatcher Illusion," Lewis \& Johnston, 1997). Participants then completed a distractor task (ranking various objects) to facilitate the emergence of an approach state following a source of uncertainty (for a review, see Proulx et al., 2012). Participants were then randomly presented with either a gain or loss-framed Asian disease problem decision (Kahneman \& Tversky, 1979), in which sure and risky choice options were counterbalanced.

Gains framing: "Imagine that the United States is preparing for the outbreak of an unusual Asian disease, which is expected to kill 600 people. Two alternative programs to combat the disease have been proposed. Assume that the exact scientific estimates of the consequences of the programs are as follows: If program A is adopted, 200 people will be saved. If program $B$ is adopted, there is a 1/3 probability that 600 people will be saved and a 2/3 probability that nobody will be saved." Losses framing: "Imagine that the United States is preparing for the outbreak of an unusual Asian disease, which is expected to kill 600 people. Two alternative programs to combat the disease have been proposed. Assume that the exact scientific estimates of the consequences of the programs are as follows: If program $A$ is adopted, 400 people will die. If program $B$ is adopted, there is a 1/3 probability that nobody will die and a 2/3 probability that 600 people will die."

Next, we recorded each participant's binary choice by asking them "Which of the two programs would you favor?". We then measured participants' "scaled risk preference" (Mandel, 2014), where we asked them "Please indicate the extent to which you would favor Program A" and "Please indicate the extent to which you would favor Program B" with a 100 point scale slider $(0=$ No Preference at All, $100=$ Extremely Prefer for A (or 'B' $))$. We 
calculated the net scaled risk preference by using the scaled preference for risky options minus the scaled preference for sure options - “-100” means full preference for sure option whereas " $+100 "$ means full preference for risky option. Consistent with the procedure of Kahnema and Tversky (1979), participants were also presented a text box and asked "Please briefly explain your rationale for your decisions". Consistent with prior research on the Asian-disease problem, we did not analyze the text answers.

At the end of the survey, participants were asked whether they were able to complete this survey in one sitting, without any distractions, and were debriefed.

\subsection{Results}

The (net) scaled risk preference ranged from -100 to 100 , with mean of $-0.22, S D=$ 54.49. The correlation of scaled and binary risk preference was .79, $p<.01$, suggesting people were consistent in their preference regardless of assessment.

To test our predictions regarding risk preference, we regressed (net) scaled risk preference onto BA (centered), framing (gains versus losses; effect coded; gains framing coded -1 , losses framing coded +1 ), expectancy violation (control versus experimental condition; effect coded; control condition coded -1 , expectancy violation condition coded +1 ), their two-way interactions and the three-way interaction into a single regression model.

\subsubsection{Main effects: Framing, Expectancy Violation, and Trait BA.}

As expected, we replicated a significant main effect of framing, $B=11.04, S E=3.81, t$ $(186)=2.89, p<.01$, such that participants were more risk-seeking under losses framing than under gains framing. However, contrary to $\mathrm{H} 1$ and $\mathrm{H} 2$, the main effect of expectancy violation was not significant, $B=1.12, S E=3.81, t(186)=0.29, p=.77$, nor was the main effect of trait BA, $B=.28, S E=.63, t(186)=0.44, p=.66$ 


\subsubsection{Interactions: Framing, Expectancy Violations, and Trait BA.}

In support of $\mathrm{H} 3$ and $\mathrm{H} 4$, there was a hypothesized three-way interaction $(B=1.74, S E$ $\left.=.63, t(186)=2.74, p<.01, R_{\text {adj }}^{2}=.08\right)$, whereby we found a significant interaction between expectancy violation and trait BA under losses framing $(b=5.81, S E=1.84, t(186)=3.15, p$ $<.01)$ and no significant interaction between expectancy violation and trait BA under gains framing $(b=-1.14, S E=1.74, t(186)=-0.66, p=.51)$. See Fig. 1 for a plot of the interactions.

We then proceeded to analyze the simple slopes for the interaction under losses framing. In the control condition, there was no relationship between risk preference and BA $(b=-2.08$, $S E=1.41, t(186)=-1.48, p=.14)$. In contrast, in the expectancy violation condition, there was a positive relationship between risk preference and BA, $(b=3.73, S E=1.19, t(186)=$ $3.13, p<.01)$. As we hypothesized, among participants high on BA $(+1 \mathrm{SD})$, those in the expectancy violation condition were more risk-seeking (preferred risky losses option) than those in the control condition $(b=37.50, S E=16.34, t(186)=2.29, p=.02<.05)$. Unexpectedly, among those participants low on BA (-1 SD), those in expectancy violation condition were relatively risk-averse (preferred sure losses option) compared to those in the control condition $(b=-32.99, S E=14.53, t(186)=-2.27, p=.02<.05)$. 


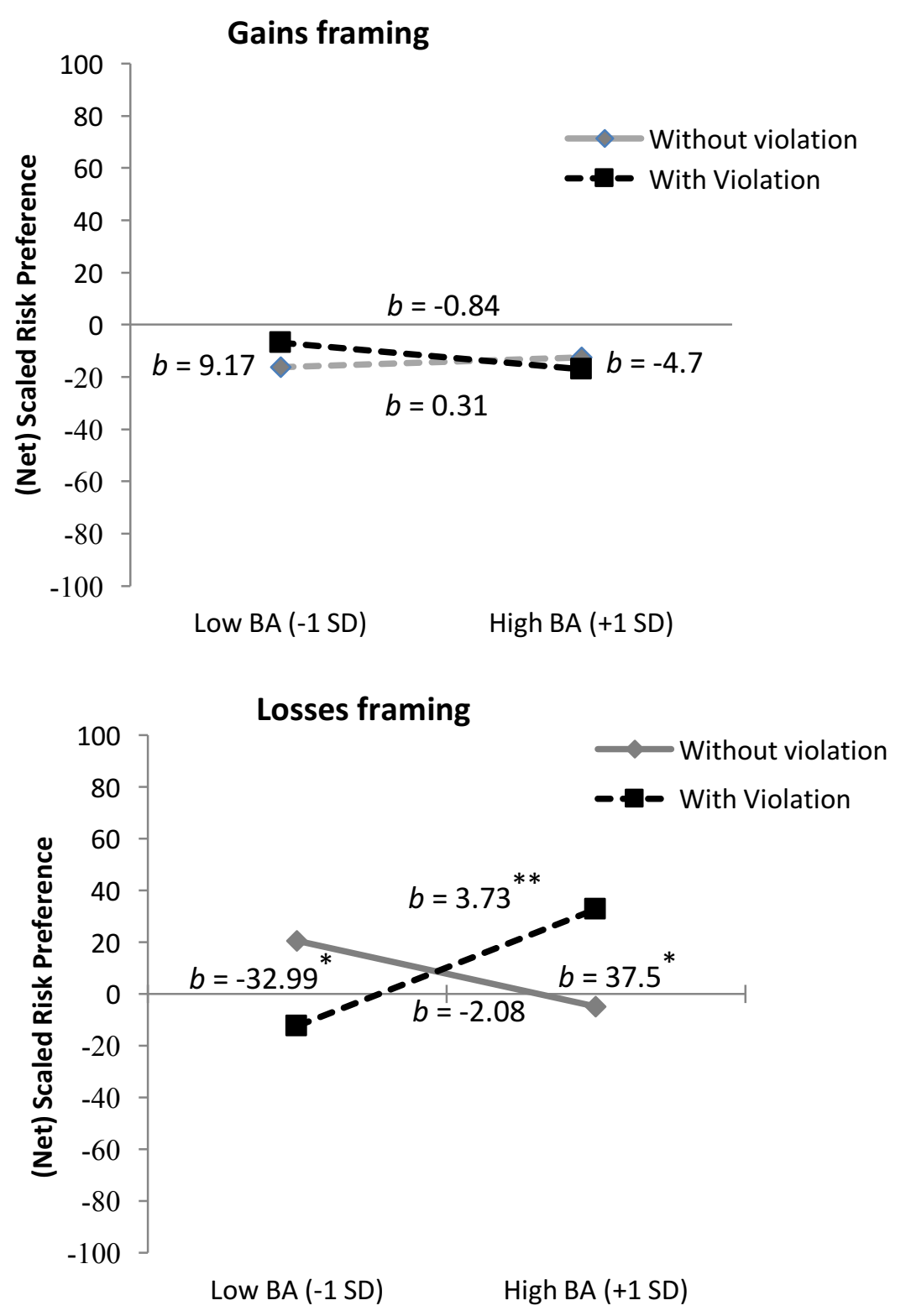

Fig. 1. Regression Lines of Interaction of Expectancy Violation $\times$ BA at Both Gains and Losses Framing in Study 1. Note. ${ }^{+} p<.10,{ }^{*} p<.05, * * p<.01$

\subsection{Discussion}

In Study 1, we examined the extent to which behavioral approach orientation (state, trait and their interaction) would affect people's risk preference in decision-making, assessed with either the gains or losses-framed Asian disease problem. While we replicated the framing effect, neither state nor trait BA, on their own, impacted people's risk preference in the gains or loss framing. However, results supported the hypothesized interaction (Hypothesis 3) 
whereby people high in trait behavioral approach favored the risky loss option more following an expectancy violation than those in a control condition, whereas the opposite occurred for people low in trait behavioral approach. Moreover, in support of Hypothesis 4, this was the case for the loss-framing, but not the gain-framing of the Asian disease problem. Under gain-framing, we did not find any significant effect of behavioral approach orientation on people's risk preference in decision-making, reinforcing the sense that people's preference for the sure option in a gains framing is relatively stable in the face of individual difference and state motivational factors.

\section{Study 2}

The results of Study 1 supported the hypothesis that expectancy violation and trait behavioral approach interact in impacting risk-seeking in losses $(\mathrm{H} 3)$ but not in gains $(\mathrm{H} 4)$ framed decisions. However, contrary to expectations, we did not find any main effect of state behavioral approach induced by expectancy violation $(\mathrm{H} 1)$ or trait behavioral approach $(\mathrm{H} 2)$ in the losses domain. Also, a surprising finding was that people low in trait BA become more risk-averse under losses framing in the expectancy violation condition as opposed to the control condition. To determine the robustness of the latter, unexpected finding, along with our main findings, we conducted a direct replication of the Study 1 in which we focus only on the losses domain.

\subsection{Method}

\subsubsection{Participants and Design.}

253 complete responses were collected online using MTurk ( $\$ 0.50$ compensation). 248 cases ( $54 \%$ female; $M_{\text {age }}=36.79, S D=13.33 ; 99.6 \%$ English native speakers) were deemed valid cases after excluding 5 participants who reported they were distracted. The design of Study 2 was trait behavioral approach (continuous) $\times$ expectancy violation (with violation versus without violation). 


\subsubsection{Procedure.}

The same measures and procedures of Study 1 were used in Study 2, except that we only focused on the losses-framed condition of the Asian disease problem. The Cronbach's alpha of the BAS scale in the current study was .84 .

\subsection{Results}

The (net) scaled risk preference ranged from -100 to 100 , with a mean of $21.37, S D=$ 49.16. The correlation of scaled and binary risk preference was $0.76, p<.01$.

As in Study 1, we analyzed the results with linear regression analysis. We regressed (net) scaled risk preference onto centered BA, expectancy violation (control versus experimental condition; effect coded; control condition coded -1 , expectancy violation condition coded +1 ) and their two-way interaction.

\subsubsection{Main effects: Expectancy Violation, Trait BA.}

As expected, we found a generally risk-seeking preference in the losses domain. The constant in Study 2 was significantly above $0(B=21.40, S E=3.10, t(244)=6.90, p<.01)$. We found a trend that participants in the expectancy violation were more risk-seeking than those in control condition $(B=4.93, S E=3.10, t(244)=1.59, p=.11)$, but this finding is marginal. As in Study 1, there was no significant main effect of trait BA $(B=.28, S E=.54, t$ $(244)=0.53, p=.60)$.

\subsubsection{Interactions: Expectancy Violation $\times$ Trait BA.}

There was a marginally significant hypothesized (H3) two-way interaction effect of expectancy violation $\times \mathrm{BA}\left(B=0.98, S E=0.54, t(244)=1.83, p=.07, R_{\text {adj }}^{2}=.01\right) . \mathrm{We}$ plotted this two-way interaction in Fig. 2.

As in Study 1, there was no relationship between risk preference and BA $(b=-0.70, S E$ $=.76, t(244)=-0.92, p=.36)$ in the control condition. Moreover, in the violation condition there was a marginally significantly positive relationship between risk preference and BA, (b 
$=1.27, \mathrm{SE}=.76, \mathrm{t}(244)=1.66, \mathrm{p}=.098)$. As a main test of our line of reasoning, we found that among participants high on trait BA $(+1 \mathrm{SD})$, those in the expectancy violation condition were more risk-seeking than those in the control condition $(b=21.22, S E=8.79, t(244)=$ $2.42, p=.02<.05)$. No effect of expectancy violation emerged among participants scoring low on trait BA (-1 SD), $b=-1.51, S E=8.79, t(244)=-0.17, p=.86$. These findings provide further support for our line of reasoning.

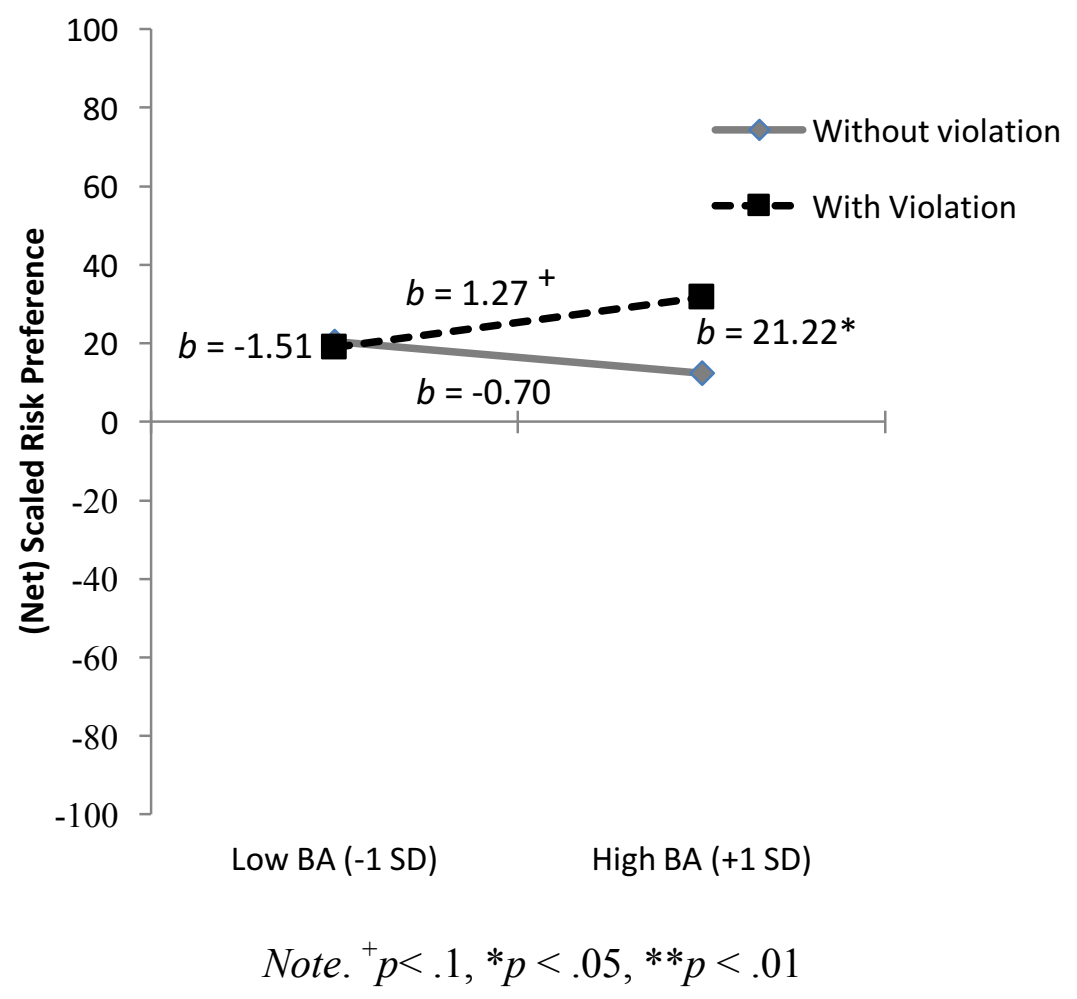

Fig. 2. Regression Lines of Interaction of Expectancy Violation $\times$ BA at Losses Framing in Study 2.

\subsection{Discussion}

Study 2 provides further evidence that the interaction of state BA and trait BA impacts people's risk preference for decisions in the losses domain. As in Study 1, people high in trait BA became more risk-seeking after exposure to an expectancy violation than after exposure to a control condition. Although the interaction obtained in Study 2 is marginally significant, the simple slopes suggest that the overall pattern of effects is reliable, further supporting our 
line of reasoning. It can also be noted that the unexpected shift from risk-seeking to riskaversion among those low in trait BA, which we observed in Study 1, did not replicate in Study 2, suggesting that this effect is not reliable.

\section{General Discussion}

The present research demonstrated that an expectancy violation can motivate people high in trait behavioral approach to be more risk-seeking under losses than they would be if they experienced no expectancy violation. Two experiments (Studies 1 and 2) demonstrated that among people high in trait behavioral approach (trait BA) risk-seeking under losses was stronger following an expectancy violation (state BA) than in the control condition. As expected, Study 1 uncovered that this was only the case for decisions framed in terms of losses, but not in terms of gains. Together, these results suggest that an expectancy violation can trigger high approach-oriented individuals to be more risk-seeking in response to losses. We suggest that the underlying motivational mechanism is that expectancy violation can induce people, especially those high in trait behavioral approach, into an approach motivational state, which would strengthen their palliative approach response to a sure loss.

Besides the "cold" cognitive models that emphasize people's perception and evaluation of information in a given decision-making situation (e.g., prospect theory; Kahneman \& Tversky, 1979), the "hot" motivational theories stress people's motivational states and personality traits in explaining people's risk-seeking under losses. The present research is consistent with the latter perspective by highlighting the combined role of motivation and personality to account for people's risk-seeking under losses. However, we advanced a new perspective, a violation-compensation angle, to explain people's risk-seeking under losses and emphasized the role of expectancy violation in triggering people's risk-seeking preference. We suggest that the discrepancy between people's desires of having no loss and the realistic possibility of loss triggered people's defensive response. Because it is not 
possible to resolve the discrepancy, the palliative approach state arising from the compensation process drove people towards more risky decision-making (Proulx et al., 2012). Therefore, an additional expectancy violation would strengthen people's risk-seeking under loss. The role of trait behavioral approach in predicting people' risk-seeking has been investigated by previous research (Suhr \& Tsanadis, 2007). However, to our knowledge, this is the first demonstration that people high on trait behavioral approach are especially likely to become risk-seeking under losses after the experience of an expectancy violation. Although we only found the strengthened risk-seeking effect under losses of an expectancy violation on high approach individuals, the present findings have broadened our understandings of people's risk-seeking behaviors in the face of losses by having brought expectancy violation into focus.

The practical implications of the present research are that people's natural tendency to take risks when faced with losses can be further exacerbated by expectation violations and their own personal tendencies in terms of their trait BA. This may help to illuminate people's sometimes irrational risk-taking tendencies, such as following the accidental loss of a family member mentioned earlier, or excessive loss-chasing in casinos. This is important, as irrational or foolish risky decisions-making under losses may bring about even more disastrous consequences. It might be speculated that excessive risk-taking can be reduced by stimulating the Behavioral Inhibition System (BIS), promoting an avoidance motivation. Moreover, we note that not all risk-taking necessarily is bad (e.g., entrepreneurship), and the present research may likewise provide tools to reduce excessive risk-aversion in people.

Our findings consistently demonstrate that trait BA is positively associated with an increase in risk-seeking in the loss domain only when there is a source of uncertainty present in the environment - in this case, expectancy violating facial features. This is to say, the combination of trait and state BA appeared to drive our effects. On their own, it did not 
appear that a state of reactive approach following trivial uncertainties is sufficient to impact risk judgments in the same manner as trivial uncertainties have been shown to impact the affirmation of committed beliefs (Proulx et al., 2012). It was also the case that trait BA, on its own, did not appear to be associated with heighted risk-preference. It could be that the absence of main effects for state or trait BA is due to methodological limitations of the present studies. For example, the relatively subtle and implicit nature of the expectancy violation used in this study (implicit visual anomaly) may not be a potent enough source of uncertainty to impact relatively complex risk assessments in the same manner as unilateral belief affirmations (Sleegers et al., 2015). Future research could explore more explicit sources of uncertainty (e.g., mortality salience) which may motivate an overall preference for risk in a loss domain. With regards to trait $\mathrm{BA}$, it could be the case those most extreme in their trait BA do display heightened risk preference, but this population was not sufficiently represented in our online sample. Future research could examine this relationship for those who deviate significantly form the mean on the trait behavioral approach scale.

\section{Conclusions}

Overall, our approach complements the cognitive and formal theories of risky choice (e.g. prospect theory, Kahniman \& Tversky, 1979) by highlighting the importance of motivational accounts (both situational and chronic) for people's risk-seeking in a losses domain. Risk preference in a losses domain is impacted by the conjunction of situational behavioral approach states and chronic behavioral approach traits. That is, high approach individuals become even more risk-seeking when they react to unexpected losses in an uncertain world where there are full of various kinds of violations. 


\section{Acknowledgments}

This research was supported in part by a grant from the Chinese Scholarship Council of People's Republic of China awarded to Xiayue Tan (No. 201307720031) and by a NWO Veni grant awarded to Travis Proulx (451-11-025). 


\section{References}

Baumeister, R.F. (2003).The psychology of irrationality: Why people make foolish, selfdefeating choices. The Psychology of Economic Decisions: Volume I: Rationality and Well-Being. Isabelle Brocas, Juan D. Carrillo (Ed.), New York, CA: Oxford University Press, p1-17.

Campbell-Meiklejohn, D. K., Woolrich, M. W., Passingham, R. E., \& Rogers, R. D. (2008). Knowing when to stop: the brain mechanisms of chasing losses. Biological psychiatry, 63(3), 293-300.

Carver, C. S., \& White, T. L. (1994). Behavioral inhibition, behavioral activation, and affective responses to impending reward and punishment: The BIS/BAS scales. Journal of Personality and Social Psychology, 67, 319-333.

Elliot, A. J., \& Thrash, T. M. (2002). Approach-avoidance motivation in personality: approach and avoidance temperaments and goals. Journal of personality and social psychology, 82(5), 804.

Gray, J. A. (1990). Brain systems that mediate both emotion and cognition. Cognition \& Emotion, 4(3), 269-288.

Jonas, E., McGregor, I., Klackl, J., Agroskin, D., Fritsche, I., Holbrook, C., Nash, K., Proulx, T., \& Quirin, M. (2014). Threat and defense: From anxiety to approach. Advances in experimental social psychology, 49, 219-286.

Kahneman, D., \& Tversky, A. (1979). Prospect Theory: An Analysis of Decision under Risk. Econometrica, 47, 263-291.

Kühberger, A. (1997). Theoretical conceptions of framing effects in risky decisions. Decision making: Cognitive models and explanations, 128-144. 
Kühberger, A., Schulte-Mecklenbeck, M., \& Perner, J. (1999). The Effects of Framing, Reflection, Probability, and Payoff on Risk Preference in Choice Tasks. Organizational Behavior and Human Decision Processes, 78, 204-231.

Lewis, M. B., \& Johnston, R. A. (1997). The Thatcher illusion as a test of configural disruption. PERCEPTION-LONDON-, 26, 225-228.

Lopes, L. L. (1987). Between hope and fear: The psychology of risk. Advances in experimental social psychology, 20, 255-295.

Mandel, D. R. (2014). Do Framing Effects Reveal Irrational Choice? Journal of Experimental Psychology, 143(3), 1185-1198.

McGregor, I., Nash, K., Mann, N., \& Phills, C. E. (2010). Anxious uncertainty and reactive approach motivation (RAM). Journal of personality and social psychology, 99(1), 133.

McNaughton, N., \& Gray, J. A. (2000). Anxiolytic action on the behavioural inhibition system implies multiple types of arousal contribute to anxiety. Journal of affective disorders, 61(3), 161-176.

Lewis, M. B., \& Johnston, R. A. (1997). The Thatcher illusion as a test of configural disruption. PERCEPTION-LONDON-, 26, 225-228.

Proulx, T., \& Inzlicht, M. (2012). Moderated disanxiousuncertlibrium: Specifying the moderating and neuroaffective determinants of violation-compensation effects. [Reply] Psychological Inquiry. 23, 386-396.

Proulx, T., Inzlicht, M., \& Harmon-Jones, E. (2012). Understanding all inconsistency compensation as a palliative response to violated expectations. Trends in cognitive sciences, 16(5), 285-291.

Scholer, A. A., Zou, X., Fujita, K., Stroessner, S.J., Higgins, E.T.(2010). When risk seeking becomes a motivational necessity. Journal of Personality and Social Psychology, $99(2), 215-231$. 
Schneider, S. L., \& Lopes, L. L. (1986). Reflection in preferences under risk: Who and when may suggest why. Journal of Experimental Psychology: Human perception and performance, 12(4), 535.

Sleegers, W. W., Proulx, T., \& van Beest, I. (2015). Extremism reduces conflict arousal and increases values affirmation in response to meaning violations. Biological Psychology, 108, 126-131.

Suhr, J. A., \& Tsanadis, J. (2007). Affect and personality correlates of the Iowa Gambling Task. Personality and Individual Differences, 43(1), 27-36.

Tversky, A., \& Kahneman, D. (1981). The framing of decisions and the psychology of choice. Science, 211, 453-458.

Wallhoff, F. (2006).Facial Expressions and Emotion Database. Retrieved from: http://www.mmk.ei.tum.de/ waf/fgnet/feedtum.html, Technische Universität München. 